\title{
SIMULATION OF SYSTEMS FOR MONITORING, ANALYSIS AND FORECASTING OF THE PRIORITY DIRECTIONS OF SCIENCE AND TECHNOLOGY
}

\author{
Tatyana Abramova ${ }^{\mathrm{a}}$ \\ National Research Tomsk State University, 634050, Tomsk, Russia
}

\begin{abstract}
The article presents the automated scheme for monitoring, analysis and forecasting based on the extended conceptual foresight model, which is a system of methods of expert evaluation of the strategic prospects of innovative development, identification of technological breakthroughs that can have maximum influence on economy and society in the medium and long-term outlook.
\end{abstract}

\section{Introduction}

Foresight is one of the tools, which allows identifying priority areas of science, technology, innovation and making a concrete action plan for their development. Foresight is a system of methods of expert evaluation of the strategic prospects of innovative development, identification of technological breakthroughs that can have maximum influence on economy and society in the medium and long-term outlook.

Herbert Wells introduced the concept of «Foresight» in 1932. In one of his public speeches, a well-known science fiction writer spoke about the need for «Foresight Professor» - a new scientific specialty aimed at studying future technological breakthroughs and search for areas of their application.

However, as a system of methods, Foresight appeared much later, during the period of 1940-1950. At that time, Foresight has already been considered as a systematic and systemic process enabling to assess future development of a subject area by means of expert evaluations. However, its methodical apparatus was quite limited. American RAND Corporation introduced a number of significant innovations, which gained wide dissemination later.

\section{History of Foresight methods}

Delphi method, a multi-level expert survey with feedback, was among the first basic Foresight methods [1]. The principle of this method is as follows: at the first stage, a large number of experts (often, several thousands of people) are invited to fill in special

\footnotetext{
${ }^{a}$ Corresponding author: tanusha-atv@mail.ru
} 
questionnaires containing questions about the prospects of certain developments, level of their adoption in the country and abroad, etc. Processed questionnaire results, including not only questions but also summarized answers of the previous stage, are directed to the same experts. Respondents have an opportunity to confirm or change their earlier answers in light of the replies of other members of their panel. In practice, the Delphi method is usually carried out in two rounds, although the number of rounds can be greater.

Other countries have used successful US experience of technology Foresight in the middle of the XX century. Based on the Delphi method, «Future Technology in Japan toward the Year 2030» project was implemented in Japan in 1971. The National Institute of Science and Technology Policy of Japan (NISTEP), one of the leading centers in the field of long-term forecasting, conducted the study [2]. Since that time, Foresight at the national level is carried out regularly in this country; currently work on the ninth long-term forecast has been completed.

Since 1980-ies, France, Sweden, Canada, Australia have carried out pioneering work on technological Foresight. During this period, a sharp increase of interest in Foresight studies has been marked. At this time, the US Ministry of Defense and Ministry of Commerce have started the Foresight project aimed to identify «critical technologies»; the Netherlands have implemented Foresight projects under the auspices of the Ministries of Economy, Education and Science; national Foresights in Germany and the UK have drawn a wide public response.

Since 1990ies, prefix «technological» is less frequently used in relation to Foresight. The last two cycles of British Foresights were no longer called «technological»; they reflected broader and more comprehensive vision for the future development. During this period, Foresight has exceeded limits of the study of science and technology; it is widely used for analysis of prospective markets. In recent years, it is applied for prediction of social processes, as well as during the process of formation of the infrastructure of national innovative systems. In fact, it is a complex prediction of development of all sides of society. It is a natural result. A modern innovative model requires developed system of professional training, high educational and cultural level of the population, scientific potential, efficient communications system, and maintenance of a high level of social stability. Today Foresight is focused on assess of prospects for socio-economic development in a global context and ensuring sustainable development in conditions of growing interdependence of the various spheres of society.

In 2000, more than 30 countries have actively used Foresight at the national level. Currently, not only Western Europe, the USA and Japan use this technique, but also a number of developing countries and countries with transition economy - the new EU members, particularly, Hungary, the Czech Republic and Poland. Over the past quarter of the century, individual developed countries and large companies have realized more than one thousand Foresight projects in total.

\section{System of Foresight methods}

Table 1 shows the main differences between Foresight and forecasting. 
Table 1. Comparative analysis of Foresight and Forecasting.

\begin{tabular}{|c|c|c|}
\hline Principle & Foresight & Forecasting \\
\hline Involvement & $\begin{array}{l}\text { Representatives of the whole } \\
\text { society participate }\end{array}$ & Conducted by scientists \\
\hline Communication & $\begin{array}{l}\text { Communication between } \\
\text { participants is important to reach } \\
\text { consensus }\end{array}$ & $\begin{array}{l}\text { Communication between } \\
\text { participants of forecast is not an } \\
\text { important factor }\end{array}$ \\
\hline Research horizon & $\begin{array}{l}\text { Usually, } 10-15 \text { years, sometimes } \\
80-90 \text { years }\end{array}$ & $\begin{array}{l}\text { Horizon is strongly dependent on } \\
\text { availability of dynamic data-series. } \\
\text { On average, less than that of } \\
\text { Foresight }\end{array}$ \\
\hline Coordination & $\begin{array}{l}\text { Evaluation of development of } \\
\text { science and technology are } \\
\text { inseparable from economic and } \\
\text { social changes }\end{array}$ & The main focus is on a subject area \\
\hline Consensus & $\begin{array}{l}\text { It determines whether there is a } \\
\text { consensus regarding subjects under } \\
\text { the study }\end{array}$ & $\begin{array}{l}\text { Presence or lack of consensus is } \\
\text { not an important factor }\end{array}$ \\
\hline Expertise & Expert methods dominate & Quantitative methods dominate \\
\hline Alternativeness & $\begin{array}{l}\text { Alternative options to achieve the } \\
\text { desired image of the future are } \\
\text { considered }\end{array}$ & Basic option is usually considered \\
\hline Systemacy & $\begin{array}{l}\text { Foresight covers not only the } \\
\text { subject area, but also its } \\
\text { environment }\end{array}$ & Forecast is made for a specific area \\
\hline Openness & $\begin{array}{l}\text { Initial data, needs, research issues } \\
\text { are still opened and can be revised } \\
\text { during the course of Foresight. The } \\
\text { results are brought to attention of } \\
\text { as many people as possible }\end{array}$ & $\begin{array}{l}\text { Initial data, themes and research } \\
\text { questions can be determined } \\
\text { beforehand }\end{array}$ \\
\hline
\end{tabular}

In general, the system of Foresight methods is constantly being developed and improved; great experience for their practical application has been accumulated over the past ten years. Today, a set of approaches covers dozens of techniques - both qualitative (interviews, literature reviews, morphological analysis, "correspondence trees», scenarios, role-plays, etc.) and quantitative (cross-impact analysis, extrapolation, modeling, analysis and forecast of methods indicators, etc.). A number of methods are of synthetic nature. They include abovementioned Delphi method, the road map, the critical technologies as well as multi-criteria analysis, patent analysis, game simulation, etc. A set of methods used in a particular project can be selected based on a variety of factors: time and resource limitations, availability of sufficient number of highly qualified experts, access to information sources and others. However, use of methods that ensure efficient work of experts is a key to success of a project.

Effectiveness of the combined use of different qualitative and quantitative methods has been confirmed. At the same time, it has became obvious that big projects devoted to the choice of technology priorities at the national level require new approaches, which can ensure obtaining of objective assessments based on quantitative analysis of empirical data - 
statistical indicators, patent statistics, bibliometric information, etc. In this connection, the idea of the «Foresight triangle» has undergone some changes. I. Miles and R. Popper [3] proposed to add one more vertex - «Evidence» and thus to turn the triangle into the rhombus (Figure 1).

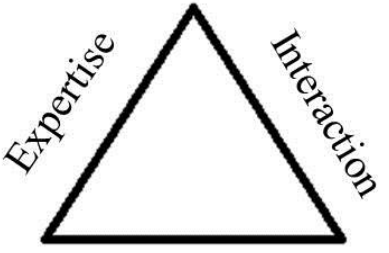

Creativity

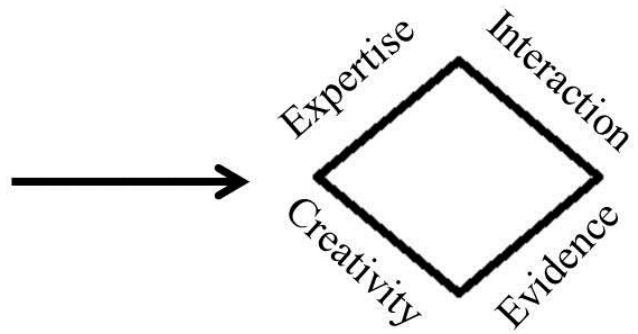

Figure 1. Conceptual transition from the Foresight - triangle to the Foresight - rhombus.

Currently, Foresight includes methods relating to different areas of science: sociology, mathematics, statistics, etc. There are four key categories of Foresight methods [4-9]:

- Expertise. In order to verify results obtained from analysis of primary sources, it is necessary to discuss trends of development of the subject areas with key experts representatives of science, business, and government organizations.

- Creativity. In order to predict unexpected turns of the future, you must add some creative elements to the analysis. Typical examples of creative methods include wild cards («random factors», «Joker») and weak signals. Essence of the wild cards method is that based on various types of information (scientific and journalistic articles, interviews with experts, etc.) specialists identify events that have a low probability of occurrence, but can have great influence on the further course of development. One of the typical examples of wild cards is an accident at the Fukushima nuclear power plant, which forced the international community to take a fresh look at the prospects of the nuclear energy development.

- Interaction. It is necessary to involve in Foresight process as many sides as possible, including representatives of science, business, government, and to ensure their ongoing dialogue on the future development. If the subject of the research affects wide sectors of society, much attention should be paid to informing people about the Foresight project and its main conclusions. This can be achieved by carrying out various kinds of workshops, panels of citizens, etc.

- Evidence. In order to get reliable Foresight results it is necessary to base on previously published materials and to study best international practice in the subject area. In addition, you can form a pool of the most qualified experts in the subject area based on patent and bibliometric analysis.

Today, Foresight is important for Russia because it is an example of a communication platform that allows wide discussion of actual problems of science and technology, development of innovative strategies with participation of all sides, and coordination of the implementation process.

Foresight makes it possible to provide adequate analysis, monitoring and forecasting development of science and technology. Foresight enables to concentrate innovative resources on the most important scientific and technical problems in the medium and longterm outlook, to prepare proposals on measures of the state scientific, technical and 
innovative policy. This allows providing authorities for innovations, science and technology of the Russian Federation with current, complete, accurate and regularly updated information in the sector of research and development. Finally, by means of Foresight, it is possible to develop innovative strategies for the industries of the Russian economy, and thereby contribute to strengthening of national competitiveness.

\section{Indicators and criteria}

Development of indicators for the experts' assessment of technological alternatives as well as selection criteria is a separate subject of study in the work. Now, we can conclude that the content of selection criteria directly depends on the nature of strategic priorities the state government. We can distinguish different «primacy» of strategic national priorities at different levels of government, i.e. the main tasks can include «support of basic science» or «improvement the life quality», «achievement of high rates of economic growth», «development of education and culture», «national defense and security». Ranking of priorities is authority of the legislative branch.

It is advisable to consider the applicability of known criteria of projects evaluation for selection (as a priority) of those technological trends that are being on the stages of development and production of a prototype. Along with this, it is necessary to explore the possibility of primary support not only to developments, which are almost ready for the market launch, but to those researches, which are on the pre-competitive stage of the life cycle, the so-called new and emerging areas of science and technology.

In order to select priority areas and critical technologies, we need to consider «critical» problems taking into account the achieved and achievable level of development of national science and technology. In this case, the «critical problem» is an innovative problem of social and economic sphere, which meets at least one of the following conditions. The problem is critical for many producers or social individuals; or it is a necessary link in the process of manufacturing of a product, which is extremely crucial for economy competitiveness; or it is a necessary link for solving a social problem, which is considered as crucial. Critical issues serve as a motive for the choice of technology.

In this case, participation of representatives of various sectors of society (government, science, industry, business, and social structures) in selection of priorities for scientific and technological development is possible. Use of discussions and surveys within formulation of the so-called «technology roadmaps» or projects of future forecasting (Foresight) seems sensible.

As for the possibility of including social technologies in the lists of priority directions and lists of critical technologies, we can conclude that there are no principle objections for lists to be close to areas such as improving quality of life, support of education and culture. For selection of social technologies, it is necessary to set criteria characterizing possibility to achieve social priorities and socio-economic goals.

We should also note that in conditions of fee-for-scientific and technical information, including patent statistics, and increasing subjectivity of expert assessments, the role of information support for selection of priorities of scientific and technological development is increasing. However, federal statistical data and sampled data are insufficient. In order to achieve completeness, accuracy, relevance of information during implementation of the project on development of lists of priority directions and critical technologies (at any level federal, regional, sectorial), it is advisable to develop a methodology and to carry out regular comprehensive patent research. This will allow following the patent situation in realization of critical technologies in Russia and abroad, including identification of «patent surge», forecasting of new technological trends, and identification of competitive situation. 
Study of the problem of development of a special technology classifier and analysis of opportunities of use of existing national, international, foreign and thematic rubricators and classifiers of scientific and technical information is one more urgent direction for further development of the methodology for selecting priorities of scientific and technological development and critical technologies.

Thus, the system of scientific priorities and critical technologies is not linear. It is a hierarchically constructed multi-level system, which changes dynamically and evolutionary in accordance with the principles of adaptation to the current socio-economic situation of the global and national scale, taking into account trends of global scientific and technological development and anticipatory predictive scenarios of improvement and development of scientific and technological potential of Russia.

All more or less successful projects of recent years are based on integrated approaches. Moreover, we can clearly see a tendency of constant complication of the system of used methods, including application of mathematical methods allowing strengthening Foresight study by scientifically grounded assessments. Such assessments allow making a more detailed scenario forecast based on quantitative evaluations of target indicators and identifying classification rules «If... then» in order to make necessary recommendations on updating the list of critical technologies. We can say that expert opinions and conclusions undergo a kind of test on the possibility to be proved by means of methods of intelligent analysis.

\section{Conclusion}

Based on the results of the study, we have proposed a conceptual model of conducting expert interviews according to a flexible system of indicators describing the technology.

At the initial stage, it includes a range of quantitative and qualitative indicators outlined by experts. At the next stages, it can be modified by other features depending on socioeconomic and political target factors. The developed automated system includes two levels, which are interconnected by analysis and forecast. Automated system, which operates in the iterative mode, is simple and does not need any comments.

In conclusion, we should note that the methodological approach based on the evidence by mathematical methods of analysis develops the technology of Foresight studies and fully integrates in it.

\section{Acknowledgements}

The author wishes to thank Tatiana B. Rumyantseva, National Research Tomsk State University, for assistance in the article preparation.

The paper was written as part of the research project No. 8.2.31.2015, carried out with the support of the Program "Research Foundation of Tomsk State University named after D.I. Mendeleev" in 2015 - 2016., grant RFBR No. 16-29-12858.

\section{References}

[1] N.C. Dalkey, O. Helmer-Hirschberg, An experimental application of the Delphi method to the use of experts RAND Report RM-727-PR (1962)

[2] NISTEP. The 8th science and technology Foresight survey - Delphi analysis. National Institute of Science and Technology Policy, Report PS-14 (2005)

URL: http://www.nistep.go.jp/HP_E/researchworks/02_foresight/index.html 
[3] R. Popper, Foresight Methodology, in L. Georghiou, J. Cassingena, M. Keenan, I. Miles, R. Popper (eds.), The Handbook of Technology Foresight ( Edward Elgar, Cheltenham, 2008),pp.44

[4] S.V.Gorbachev, V.I. Syryamkin, M.V. Syryamkin, Intellectual Foresight-Forecast of Scientific and Technological Development of a State (LAMBERT Academic Publishing, Saarbrucken, 2012)

[5] A.Yu. Apokin, D.R. Belousov, Foresight and STI Governance 3, 12 (2009)

[6] P.M. Derevyanko, Proceeding of management and economy in creative activity of young scientists (UNECON), 98 (2005)

[7] D.V. Shashev, S.V. Shidlovskiy, V.I. Syriamkin, A.V. Yurchenko, IOP Conference Series: Materials Science and Engineering 81, 012101 (2014) doi: 10.1088/1757899X/81/1/012101

[8] J.J. Buckley, Fuzzy Sets and Systems 21, 257, (1987) doi: 10.1016/01650114(87)90128-X

[9] A. Kofman, H. H. Aluha, Introduction of fuzzy set theory in the management of enterprises (Higher School, Minsk, 1992 ) 